\title{
How accurately can we predict synthesis cross-sections of superheavy elements?
}

\author{
David Boilley, ${ }^{1,2, *}$ Bartholomé Cauchois,,${ }^{1,2}$ Hongliang Lü, ${ }^{1,2}$ Anthony Marchix, ${ }^{3}$ Yasuhisa Abe, ${ }^{4}$ and Caiwan Shen ${ }^{5}$ \\ ${ }^{1}$ GANIL, CEA/DRF-CNRS/IN2P3, Caen, France \\ ${ }^{2}$ Normandie Université, Unicaen, Caen, France \\ ${ }^{3}$ Irfu-CEA, Université Paris-Saclay, Gif-sur-Yvette, France \\ ${ }^{4} R C N P$, Osaka University, Ibaraki-shi, Japan \\ ${ }^{5}$ School of Science, Huzhou University, Huzhou, China
}

Synthesis of superheavy elements beyond oganesson is facing new challenges as new target-projectile combinations are necessary. Guidance from models is thus expected for future experiments. However, hindered fusion models are not well established and predictions in the fission barriers span few MeVs. Consequently, predictions are not reliable. Strategies to constrain both fusion hindrance and fission barriers are necessary to improve the predictive power of the models. But, there is no hope to get an accuracy better than one order of magnitude in fusion-evaporation reactions leading to superheavy elements synthesis.

Keywords: superheavy elements; nuclear reactions; uncertainty analysis.

2 After the recent successes which lead to fill-up the last line 3 of Mendeleev's periodic table [1], the synthesis of superheavy 4 elements is facing new challenges. The heaviest synthetic el5 ements have all been created in collisions of two heavy nu6 clei. For a historical review, see Ref. [2]. However, one has 7 to find new target-projectile combinations to extend further 8 the periodic table. For cold fusion reactions, the expected 9 cross-sections are too low with present facilities to expect to 10 synthesise a new element in a reasonable timeframe. And, for 1 hot fusion reactions, there is no available target in sufficient 12 quantity anymore to be associated with the ${ }^{48} \mathrm{Ca}$ beam. Heav${ }_{3}$ ier projectiles must be used. Thus, one has to find new opti${ }_{14}$ mum target-projectile combinations. Guidance from models 15 is expected to optimise future experiments, and various pre${ }_{16}$ dictions have been continuously published in the scientific lit17 erature. Accurate predictions are necessary as a small change 18 in the cross-section could mean months of beam time for an 19 experiment.

However, a direct comparison shows that predictions dis21 agree with each other $[3,4]$ even if the models can reproduce 2 existing data. One of the reasons is the so-called fusion hin${ }_{23}$ drance, i.e. the strong reduction in the fusion cross-section 24 with respect to what is calculated by a simple extrapolation 25 of fusion models with light nuclei. Its origin is well under${ }_{26}$ stood and it is now widely acknowledged that the dynamical 27 trajectory for the fusing system must pass over a conditional ${ }_{28}$ saddle point in a multidimensional space in order to form a 29 compound nucleus, in contrast to light systems for which the 30 conditional saddle point lies outside the point of hard contact 31 in heavy-ion reactions. Dissipation also plays a crucial role 32 to understand the fusion hindrance. But, there is no consenзз sus on the dynamical models, leading to large discrepancies 34 in predictions.

The total fusion-evaporation cross-section is a combination 36 of three steps described by three different models, namely the

\footnotetext{
* Corresponding author, boilley@ ganil.fr
}

37 capture cross-section that brings the two nuclei in contact, 38 the formation probability to reach the compound shape and 39 the survival probability accounting for neutron evaporation 40 from this excited compound nucleus which competes with the 41 predominant fission decay mode:

$$
42
$$$$
\sigma_{E R}=\sigma_{c a p} \times P_{C N} \times P_{s u r} .
$$

${ }_{43}$ Capture and survival phases can be described by extrapolated 44 models used for the fusion of light nuclei. They are supposed 45 to be the best known parts of the reaction. Thus, the physics ${ }_{46}$ used to estimate $\sigma_{c a p}$ and $P_{\text {sur }}$ is well established; however, 47 parameters entering the models are not well constrained lead48 ing to uncertainties in the predictions.

The formation probability that is very specific to heavy so ions collisions, is responsible for the hindrance phenomenon. 51 There is no consensus on the dynamical model nor on the pa52 rameters. The physics used to describe the formation phase 53 faces several open questions, and, as pointed out in Refs. $54[3,4], P_{C N}$ calculated by various models spans two or three 55 orders of magnitude. See Fig. 1. Thus, there is no hope to 56 produce reliable predictions without assessing the formation 57 step.

References [3, 4] also show that once multiplied by $\sigma_{c a p}$ 59 and $P_{\text {sur }}$, all models converge to experimental data. This 60 means that the uncertainties in $\sigma_{c a p}$ and $P_{\text {sur }}$ are large enough 61 to compensate the discrepancies of the various formation 62 models and can be adjusted to get experimental data $[5,6]$.

63 Thus, to improve the predictive power of the models, we 64 also have to find ways to reduce uncertainties in $\sigma_{c a p}$ and ${ }_{65} P_{\text {sur }}$. Capture cross-section can be directly measured and the 66 models are well constrained. Discrepancies between two rea67 sonable models are lower than an order of magnitude [5]. Re68 garding the survival probability, the decay of the compound 69 nucleus formed in the collision is dominated by fission. This 70 means that a small change in the fission width will not affect 71 much the fission probability but will induce great variations 72 of the survival probability. Therefore, it is not a surprise that 73 the uncertainty analysis performed in Refs. [5, 6] has led to 74 pin down the fission width as a key parameter that needs to 75 be assessed in order to improve the predictive power of the 76 models. 


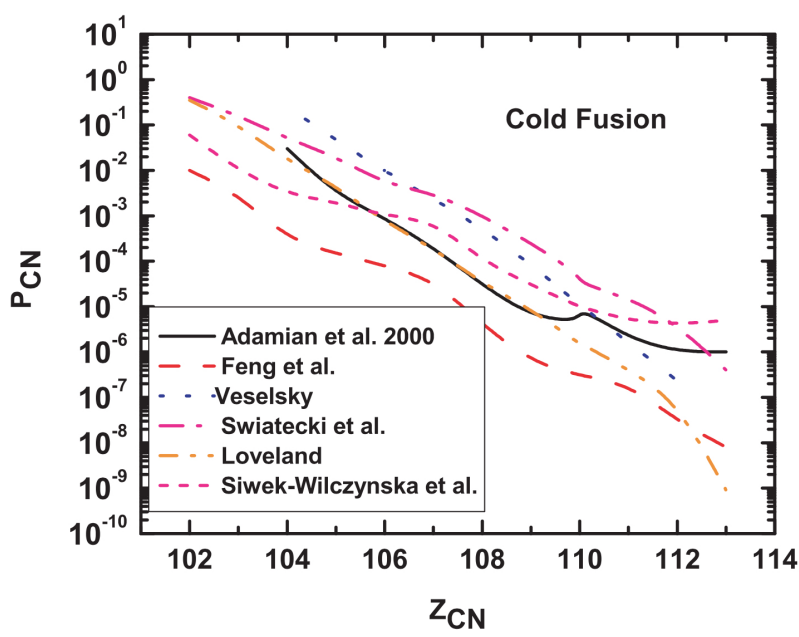

Fig. 1. Formation probability for cold fusion reactions as a function of the charge of the compound nucleus calculated by various models. Figure reproduced from Ref. [3]. See references therein for the models.

77 In this article, we shall focus on the uncertainty analysis of
78 the survival probability and propose strategies to constrain the 79 formation probability that accounts for the fusion hindrance.

\section{UNCERTAINTIES IN THE SURVIVAL PROBABILITY}

\section{A. Various predictions}

82 Compound nuclei formed during the collision decay ${ }_{83}$ through neutron evaporation or fission. Thus, the 1n survival 84 probability is nothing else than a branching ratio

$$
{ }_{85} \quad P_{1 n}=\frac{\Gamma_{n}}{\Gamma_{n}+\Gamma_{f}},
$$

86 where $\Gamma_{n}$ and $\Gamma_{f}$ stand for the neutron evaporation width and ${ }_{138}$ 87 the fission width respectively. The latter dominates. They can 88 be calculated by a standard statistical model; see e.g. Ref. [7]. ${ }_{89}$ Uncertainty in the survival probability is easily deduced from 90 the uncertainties in the widths, using the usual propagation 91 formula:

$$
\left[\frac{u\left(P_{1 n}\right)}{P_{1 n}}\right]^{2}=\left[\frac{\Gamma_{f}}{\Gamma_{n}+\Gamma_{f}}\right]^{2}\left(\left[\frac{u\left(\Gamma_{n}\right)}{\Gamma_{n}}\right]^{2}+\left[\frac{u\left(\Gamma_{f}\right)}{\Gamma_{f}}\right]^{2}\right) .
$$

145 rier of about $0.5 \mathrm{MeV}$. Another estimate based on a Bayesian

92

(3) ${ }_{146}$ analysis of a DFT model leads to a similar value for the un93 Here, we have assumed that $\Gamma_{n}$ and $\Gamma_{f}$ are independent from ${ }_{147}$ certainty in the fission barrier of ${ }^{240} \mathrm{Pu}$ [11].

94 each other. When the ratio $\Gamma_{f} / \Gamma_{n}$ is large, the relative uncer- ${ }_{148}$ All these estimates rely on a fitting procedure on nuclear 95 tainty in the survival probability reaches its maximum value. ${ }_{149}$ masses and give an uncertainty proportional to the RMS of ${ }_{96}$ This is very intuitive: as the fission decay mode dominates, 150 the fit [12]. Whatever the model, this RMS has remained over 97 evaporation events are very rare. Thus, a small change in the 151 few hundreds of keV this last decade. There is little hope to ${ }_{98}$ fission width will affect much the survival probability.

Uncertainty in the neutron evaporation width and in the fis- ${ }_{153}$ the fission barrier for a given model will also remain at few 100 sion width contributes equally. However, $\Gamma_{n}$ mainly depends 154 hundreds of keV.

101 on the neutron separation energy, whereas the fission width 155 Constraint from experiments is possible although direct 102 mainly depends on the fission barrier, the damping energy of ${ }_{156}$ measurement of the fission barrier is not an easy task. The

103 the shell correction energy and the friction coefficient when 106 tainties in the fission width and then, survival probability. As 107 shown in Refs. [5, 6], the fission barrier is the dominating 108 quantity among these three parameters.

109 Fission barriers can be calculated by various microscopic 10 or macroscopic-microscopic models, while the other two pa112 data. Comparison between different calculations [8, 9] has 113 shown that predictions in fission barriers, which is the most 14 sensitive parameter, span few MeV. Consequently, calculated 116 tude.

Even if one restricts the comparison to fission barriers that 19 span $1.5 \mathrm{MeV}$ and can induce two orders of magnitude differ20 ences in the fusion-evaporation cross-sections [5, 6]. In one 21 case, the fission barriers were estimated by the old method,

124 where $B_{L D M}$ is the fission barrier calculated with a Liquid-Model and $\Delta E_{\text {shell }}$ is the shell correction energy at the 127 ers calculated directly by the same macroscopic-microscopic

130 part of the reaction leading to the synthesis of superheavy el131 ements, the ambiguities in the fission barrier lead to discrepancies that span several orders of magnitude. This is more 134 understood.

35 Thus, reliable predictions require accurate fission barrier 136 predictions. How well can we predict such a sensitive param137 eter?

39 Assuming that the model predicting the fission barrier is 141 duce uncertainties in the calculated physical quantities. There 142 are few uncertainty analyses of the theoretical evaluation of 143 the fission barrier. In Ref. [10], a very simple micro-macro 144 model was used and leads to an uncertainty in the fission bar. 
157 heaviest nucleus which fission barrier has ever been measured 209 the cross-section due to the fusion hindrance to the synthesis ${ }_{158}{ }^{2}{ }^{254}$ No using the gamma multiplicities. Uncertainty at spin 210 of superheavy elements. For a review, see Ref. [22].

${ }_{159} 0$ is estimated to be about $0.9 \mathrm{MeV}$ [13-15]. This value is 211 Although dissipation plays a crucial role in understanding 160 extrapolated from measurements at higher spins. The fission 212 the fusion hindrance [23], associated fluctuations were not in${ }_{161}$ barrier can also be extracted by inverting a statistical decay ${ }_{213}$ cluded so far. The Langevin equation that has been used ex162 model, namely the Kewpie2 code [7]. The result is model 214 tensively to study the fluctuation-dissipation dynamics [24] ${ }_{163}$ dependent and one has to be careful when using the value ${ }_{215}$ has been adopted to study the dynamical diffusion over the 164 in another model. In particular, the extracted fission barrier ${ }_{216}$ conditional saddle that lies between the contact and the com${ }_{165}$ depends on the value of the other parameters such as the fric- ${ }_{217}$ pound configurations [25-29]. This leads to more realistic 166 tion coefficient. An analysis based on Bayesian inference was ${ }_{218}$ dependence of the fusion cross section as a function of energy ${ }_{167}$ performed to deduce the fission barrier from invented exper- 219 and a better estimate of the extremely low cross-sections.

${ }_{168}$ imental data [16]. The uncertainty in the barrier depends on ${ }_{220}$ Very recently, we showed that the initial condition of the ${ }_{169}$ the experimental uncertainty and the number of data points. ${ }_{221}$ formation step slips due to the elimination of the fast vari170 Values range from $34 \mathrm{keV}$ for quite accurate data to $0.4 \mathrm{MeV}$; 222 ables in a multidimensional description and this affects sig171 see Ref. [16] for details.

223 nificantly the formation probability [30].

Consequently, it seems that there is an incompressible ${ }_{224}$ This point of view is challenged by so-called DNS model 173 value of the uncertainty in the fission barrier that cannot be 225 that was developed later. It is based on a very different con174 overcome. Although less important, other parameters also $226 \mathrm{cept}$, assuming a frozen configuration and exchange of nucle175 have large uncertainties. The friction coefficient is not better ${ }_{227}$ ons [31-33]. Thus, it is contradictory with the models men176 known than 20 years ago. The damping energy of the shell ${ }_{228}$ tioned above based on the evolution of collective degrees of 177 correction energy has not been carefully investigated since 229 freedom.

178 the Ignatyuk's prescription [17] in 1975.

230 Although progress has been made in understanding the fu179 Because of these uncertainties in key parameters together ${ }_{231}$ sion hindrance, there is still no consensus on the dynamical 180 with the amplification effect due to the fact that we want to 232 treatment. Thus, it is almost impossible to perform an uncer${ }_{181}$ estimate the cross-section of rare events, it is hardly conceiv- 233 tainty analysis in such conditions. Moreover, many quanti182 able to produce predictions with an accuracy lower than about ${ }_{234}$ tative ambiguities remain. What is the barrier height of the 183 one order of magnitude for cold fusion reactions. It is more ${ }_{235}$ conditional saddle that has to be overcome? It is clear that a 184 for hot fusion reactions as we need fission barriers of several ${ }_{236}$ small change in its value will have a great impact on the for185 isotopes.

\section{NECESSITY TO CONSTRAIN THE FUSION HINDRANCE}

188 189 step responsible for the hindrance of the fusion, there is no 190 consensus on the model.

\section{A. Common understanding of the phenomenon}

192 Understanding and modelling fusion hindrance is a long 193 standing problem. Back in the 1960s, the first predictions 195 [18]. Nix and Sierk showed for symmetric reactions that the 250 probability that is also not well constrained as explained in 196 dynamical trajectory for the fusing system must pass over a ${ }_{251}$ the previous section. To get rid of the decay step, one should 197 conditional saddle point in a multidimensional space in or- 252 focus the step before in the reaction, i.e, the fusion cross198 der to form a compound nucleus, in contrast to light systems 253 section.

199 for which the conditional saddle point lies outside the point ${ }_{254} \quad$ The channel competing with the formation is the so-called 200 of hard contact in heavy-ion reactions [19]. Later, Świątecki 255 quasifission process. It consists in reseparation into two frag$201[20,21]$ first introduced a dynamical model that broke with 256 ments without reaching the compound state. On an experi202 the idea that the capture process can be understood in terms 257 mental point of view, of course, understanding the competi${ }_{203}$ of the static interaction of two spheres. The model empha- 258 tion between quasifission and fusion is very important. How204 sises the role of rapid dynamical deformations away from the 259 ever, it is very difficult to distinguish the quasifission frag205 configuration of two spheres in contact. It also includes dis- 260 ments from the fission ones as they have similar mass distri206 sipation that leads to the requirement of even higher energies 261 butions. The two processes differ by their time scale and thus 207 to fuse. Soon, experimental evidences confirmed the model 262 angular distribution [34]. Consequently, one lacks of reliable 208 and a lot of efforts were devoted to quantify the reduction in 263 experimental fusion cross-sections. 
264 One idea is to consider that reaction dynamics models must 292 els might simply be wrong. For example, large fission barri265 strive to reproduce experimental data on quasifission. How- 293 ers appearing in the comparison of Refs. [8, 9] would mean 266 ever, constraining models on the dominating channel leads 294 almost stable nuclei that would be easy to synthesise as the 267 to unavoidable uncertainties. And, as for fission-evaporation 295 cross-section would benefit from the large survival probabil268 competition, these uncertainties in quasifission modelling 296 ity. Second, assuming that the model is correct, we can per269 will have a large impact on the rare fusion events. Thus, re- 297 form an uncertainty analysis of the predictions in order to esti270 producing quasifission data will definitively help to constrain 298 mate the dispersion of the results due to the lack of constraints 271 models but might not allow to get a better agreement in the 299 on many parameters. Such an analysis is too optimistic as it 272 formation probability predictions.

300 assumes that the model is correct. Therefore, it is not pos273 Alternatively, hindrance could be constrained by compar- 301 sible to apply it to the whole reaction as there are still too 274 ing reactions leading to the same compound nucleus, one be- 302 many open questions on the physics of the formation phase. 275 ing hindered and the other not, in order to get rid of the am- 303 However, for the sole survival step, there is no hope to get 276 biguities in the decay phase of the reaction. Such studies, 304 predictions more accurate than an order of magnitude. This is 277 currently under development, should naturally include uncer- 305 a very pessimistic finding in the case of superheavy elements 278 tainty analysis. 306 that are produced in handful numbers.

307 If the absolute value of the predicted cross-sections can308 not be accurately estimated, the ratio between two values will 309 benefit from the strong correlation between them. This could 310 be cross-sections at different energies or with different target-

\section{CONCLUSION}

280 Although it is a difficult task, predictions of reaction cross281 sections are useless without an uncertainty analysis [35]. 282 Moreover, uncertainty analysis is a powerful tool to built up a 283 hierarchy of the various sloppy points of a model. This leads 284 to show that predictions of superheavy production cross- 315 285 sections mainly suffer from the large discrepancies in the hin286 drance phenomenon modelling and fission barriers that can 3 287 both affect the results by orders of magnitude. 311 projectile combinations. Thus, the predicted trends would be 312 correct. However, estimating the uncertainty in the ratio re313 quires a careful evaluation of the covariances. This will be 4 one of the priorities of our future research.

\section{ACKNOWLEDGEMENTS}

Part of the work is supported by the NSF of China (Grant 317 Nos. 11747312, U1732138, 11790325, 11790323) and the

There are two ways to estimate the accuracy of predictions. ${ }_{318}$ Joint International Laboratory between France and Japan 289 First is a comparison between various models. This leads ${ }_{319}$ (LIA FJNSP). We thank the RCNP of Osaka University, 290 to an estimate of the error in modelling. However, such a 320 GANIL and Huzhou University for their warm hospitality and 291 comparison can appear to be too pessimistic as some mod- 321 supports.

[1] L. Öhrström and J. Reedijk, Names and symbols of the ele- 347 ments with atomic numbers 113, 115, 117 and 118 (IUPAC 348 Recommendations 2016). Pure Appl. Chem. 88, 1225 (2016). 349 doi: 10.1515/pac-2016-0501.

[2] P. Armbruster and G. Müzenberg, An experimental paradigm 351 opening the world of superheavy elements. Eur. Phys. J. H 37, 352 237 (2012). doi: 10.1140/epjh/e2012-20046-7

[3] R. S. Naik, W. Loveland, P. H. Sprunger, A. M. Vinodkumar, 354 D. Peterson, C. L. Jiang, S. Zhu, X. Tang, E. F. Moore, and 355 P. Chowdhury, Measurement of the fusion probability $P_{C N} 356$ for the reaction of ${ }^{50} \mathrm{Ti}$ with ${ }^{208} \mathrm{~Pb}$. Phys. Rev. C76, 054604357 (2007). doi: 10.1103/PhysRevC.76.054604.

[4] W. Loveland, An experimentalist's view of the uncertainties in 359 understanding heavy element synthesis. Eur. Phys. J. A51, 120360 (2015). doi: 10.1140/epja/i2015-15120-2.

[5] H. Lü, Synthesis of Super-Heavy Elements: Role of Uncer- 362 tainty Analysis in Theoretical Modeling. Ph.D. Thesis, Nor- 363 mandie Université, Caen, France (2015) hal: tel-01235448. 364

[6] H. Lü, D. Boilley, Y. Abe, and C. Shen, Synthesis of super- 365 heavy elements: Uncertainty analysis to improve the predictive 366 power of reaction models. Phys. Rev. C94, 034616 (2016). doi: 367 10.1103/PhysRevC.94.034616.

[7] H. Lü, A. Marchix, Y. Abe and D. Boilley, KEWPIE2: 369 A cascade code for the study of dynamical decay of ex- 370 cited nuclei. Comp. Phys. Comm. 200, 381 (2016). doi: 371 10.1016/j.cpc.2015.12.003.

[8] A. Baran, M. Kował, P.-G. Reinhard, L. M. Robledo, A. Staszczak, and M. Warda, Fission barriers and probabilities of spontaneous fission for elements with $Z \geq 100$. Nucl. Phys. A944, 442 (2015). doi: 10.1016/j.nuclphysa.2015.06.002.

[9] F.P. Heßberger, Spontaneous fission properties of superheavy elements. Eur. Phys. J. A 53, 75 (2017). doi: 10.1140/epja/i2017-12260-3

[10] B. Cauchois, Uncertainty analysis: Towards more accurate predictions for the synthesis of superheavy nuclei. Ph.D. Thesis, Normandie Université, Caen, France (2018). HAL: tel01885168

[11] J. D. McDonnell, N. Schunck, D. Higdon, J. Sarich, S. M. Wild, and W. Nazarewicz, Uncertainty Quantification for $\mathrm{Nu}-$ clear Density Functional Theory and Information Content of New Measurements. Phys. Rev. Lett. 114, 122501 (2015). doi: 10.1103/PhysRevLett.114.122501

[12] B. Cauchois, H. Lü, D. Boilley, and G. Royer, Uncertainty Analysis of the nuclear Liquid Drop Model. Phys. Rev. C 98, 024305 (2018). doi: 10.1103/PhysRevC.98.024305

[13] G. Henning, A. Lopez-Martens, T.L. Khoo, D. Seweryniak, M. Alcorta, M. Asai, B. B. Back, P. Bertone, D. Boilley, M. P. Carpenter, C. J. Chiara, P. Chowdhury, B. Gall, P. T. Greenlees, G. Gurdal, K. Hauschild, A. Heinz, C. R. Hoffman, R. V. F. Janssens, A. V. Karpov, B. P. Kay, F. G. Kondev, S. Lakshmi, 
T. Lauristen, C. J. Lister, E. A. McCutchan, C. Nair, J. Piot, D. 416 [23] J.P. Błocki, H. Feldmeier and W.J. Świạtecki, Dynamical hinPotterveld, P. Reiter, N. Rowley, A. M. Rogers, and S. Zhu, 417 Exploring the stability of super heavy elements: First Mea- 418 surement of the Fission Barrier of ${ }^{254}$ No. EPJ Web of Conf. 419 66, 02046 (2014). doi: 10.1051/epjconf/20146602046

4] Greg Henning, T. L. Khoo, A. Lopez-Martens, D. Seweryniak, 421 M. Alcorta, M. Asai, B. B. Back, P. F. Bertone, D. Boilley, M. 422 P. Carpenter, C. J. Chiara, P. Chowdhury, B. Gall, P. T. Green- 423 lees, G. Gürdal, K. Hauschild, A. Heinz, C. R. Hoffman, R. V. 424 F. Janssens, A. V. Karpov, B. P. Kay, F. G. Kondev, S. Lakshmi， 425 T. Lauritsen, C. J. Lister, E. A. McCutchan, C. Nair, J. Piot, D. 426 Potterveld, P. Reiter, A. M. Rogers, N. Rowley, and S. Zhu, Fis- 427 sion Barrier of Superheavy Nuclei and Persistence of Shell Ef- 428 fects at High Spin: Cases of ${ }^{254}$ No and ${ }^{220}$ Th. Phys. Rev. Lett. 429 [27] 113, 262505 (2014). doi: 10.1103/PhysRevLett.113.262505 430

[15] A. Lopez-Martens, G. Henning, T.L. Khoo, D. Seweryniak, 431 M. Alcorta, M. Asai, B.B. Back, P. Bertone, D. Boilley, M.P. 432 Carpenter, C.J. Chiara, P. Chowdhury, B. Gall, P.T. Green- 433 lees, G. Gurdal, K. Hauschild, A. Heinz, C.R. Hoffman, R.V.F. 434 Janssens, A.V. Karpov, B.P. Kay, F.G. Kondev, S. Lakshmi, 435 T. Lauristen, C.J. Lister, E.A. McCutchan, C. Nair, J. Piot, D. 436 Potterveld, P. Reiter, N. Rowley, A.M. Rogers, and S. Zhu, Sta- 437 bility and synthesis of superheavy elements: Fighting the bat- 438 tle against fission - example of ${ }^{254}$ No. EPJ Web of Conf. 131, 439 03001 (2016). doi: 10.1051/epjconf/201613103001

16] Hongliang Lü and David Boilley, Modelling with uncertainties: 441 The role of the fission barrier. EPJ Web of Conferences 62, 03002 (2013). doi: 10.1051/epjconf/20136203002

17] A.V. Ignatyuk, G. Smirenkin, and A. Tishin, Phenomenological 444 description of energy dependence of the level density parame- 445 ter. Yad. Fiz. 21, 485 (1975)

18] T. Sikkeland, Synthesis of nuclei in the region of $Z=126$ and 447 $N=184$. Arkiv för Fysik 36, 539 (1967).

19] J. Rayford Nix and Arnold J. Sierk, Calculation of compound- 449 nucleus cross sections for symmetric very-heavy-ion reactions. 450 Phys. Rev. C15, 2072 (1977). doi: 10.1103/PhysRevC.15.2072 451

[20] W.J. Świątecki, The Dynamics of Nuclear Coalescence or Re- 452 separation. Physica Scripta 24, 113 (1981). doi: 10.1088/0031- 453 8949/24/1B/007 drance to compound-nucleus formation in heavy-ion reactions. Nucl. Phys. A 459, 145 (1986). doi: 10.1016/03759474(86)90061-8

[24] Y. Abe and S. Ayik and P.-G. Reinhard and E. Suraud, On the stochastic approaches of nuclear dynamics. Phys. Rep. 275, 49 (1996). doi: 10.1016/0370-1573(96)00003-8

[25] Y. Abe, D. Boilley, B. G. Giraud and T. Wada, Diffusion over a saddle with a Langevin equation. Phys. Rev. E61, 1125 (2000). doi: 10.1103/PhysRevE.61.1125

[26] C. Shen, G. Kosenko and Y. Abe, Two-step model of fusion for the synthesis of superheavy elements. Phys. Rev. C 66, 061602(R) (2002). doi: 10.1103/PhysRevC.66.061602

[27] D. Boilley, Y. Abe and Jing-Dong Bao, Inverse Kramers formula and fusion dynamics of heavy ions. Eur. Phys. J. A 18 627 (2003). doi: 10.1140/epja/i2003-10088-0

[28] W.J. Świạtecki, K. Siwek-Wilczyńska and J. Wilczyński, Fusion by diffusion. Acta Phys. Pol. B 34, 2049 (2003). actaphys.uj.edu.pl/

[29] W.J. Świạtecki, K. Siwek-Wilczyńska and J. Wilczyński, Fusion by diffusion. II. Synthesis of transfermium elements in cold fusion reactions. Phys. Rev. C 71, 014602 (2005). doi: 10.1103/PhysRevC.71.014602

[30] D. Boilley, Y. Abe, B. Cauchois, H. Lü and C. Shen, Elimination of fast variables and initial slip: a new mechanism to the fusion hindrance in heavy ions collisions. Submitted.

[31] N.V. Antonenko, E.A. Cherepanov, A.K. Nasirov, V.P. Permjakov, and V.V. Volkov, Competition between complete fusion and quasi-fission in reactions between massive nuclei. The fusion barrier. Phys. Lett. B 319, 425 (1993). doi: 10.1016/03702693(93)91746-A

[32] G.G. Adamian, N.V. Antonenko, W. Scheid, and V.V. Volkov, Treatment of competition between complete fusion and quasifission in collisions of heavy nuclei. Nucl. Phys. A 627, 361 (1997). doi: 10.1016/S0375-9474(97)00605-2

[33] G.G. Adamian, N.V. Antonenko, W. Scheid, and V.V. Volkov, Fusion cross sections for superheavy nuclei in the dinuclear system concept. Nucl. Phys. A 633, 409 (1998). doi: 10.1016/S0375-9474(98)00124-9

[21] W.J. Świątecki, The dynamics of the fusion of two nuclei. Nucl. 455 [34] D. Hinde, Fusion and Quasifission in Superheavy Element SynPhys. A 376, 275 (1982). doi: 10.1016/0375-9474(82)90065-3 456

[22] K.-H. Schmidt and W. Morawek, The conditions for the syn- 457 [35] Editors, Editorial: Uncertainty Estimates. Phys. Rev. A83, thesis of heavy nuclei. Rep. Prog. Phys. 54, 949 (1991). doi: 458 $10.1088 / 0034-4885 / 54 / 7 / 002$ 